\title{
Renin-angiotensin system blockade and pleiotropic cardiovascular effects: the novel angiotensin receptor blocker azilsartan
}

\author{
Ahmet Adiyaman, Ismail Adiyaman and Arif Elvan \\ Hypertension Research (2014) 37, 395-397; doi:10.1038/hr.2014.29; published online 27 February 2014
}

$\mathrm{T}$ The renin-angiotensin system (RAS) is a major regulatory system that has both cardiovascular and renal functions. RAS activation results in elevated concentrations of angiotensin-II (the principal effector peptide), which has been implicated in numerous conditions, including hypertension, heart failure, renal failure, cardiac arrhythmias, myocardial infarction and stroke. Thus, RAS inhibition has a central role in the prevention of cardiovascular disease. Multiple clinical trials over the past decades have confirmed that inhibiting the RAS reduces cardiovascular complications and mortality. ${ }^{1}$

Renin catalyzes the conversion of angiotensinogen to angiotensin-I. Angiotensin-converting enzyme (ACE) cleaves angiotensin-I to form angiotensin-II (Figure 1). Angiotensin-II acts via specific receptors, of which angiotensin-II receptor 1 (AT1) and receptor 2 (AT2) are well known. The pathologic effects of angiotensin-II are merely through AT1, which effectuates vasoconstriction, sodium retention, aldosterone secretion, increased sympathetic activity, fibrosis, cellular proliferation, superoxide formation, inflammation and thrombosis. Conversely, AT2 effectuates potentially beneficial effects, such as vasodilatation and antiproliferation. Some studies, however, have suggested that the AT2 can also have detrimental effects, such as cardiac hypertrophy and inflammation. ${ }^{2}$

This classical view of the RAS has been expanded by multiple new pathways. It is

Dr A Adiyaman and Dr A Elvan are at Isala Klinieken, Department of Cardiology, Zwolle, The Netherlands; Dr I Adiyaman is at Medwatch, Nijmegen, The Netherlands

E-mail: aadiyaman@hotmail.com now recognized that non-ACE pathways, such as chymase, cathepsin-G and chymostatin-sensitive-angiotensin-II-generating enzyme, can form angiotensin-II. Furthermore, ACE2, which is present in the heart and kidney, can mediate the conversion of angiotensin-I to angiotensin 1-7. Angiotensin 1-7 is known for its beneficial effects by promoting nitric oxide formation and counteracting the effects of angiotensin-II. ACE inhibitors (ACE-i) inhibit angiotensin-II formation; however, they accumulate bradykinin, which has additional antihypertensive, antiproliferative and anti-thrombogenic effects. Some of the beneficial cardiovascular effects of ACE-i are thought to occur through this mechanism. In addition, AT1 promotes aldosterone formation, which exacerbates the deleterious effects of angiotensin-II by promoting endothelial dysfunction, thrombosis, myocardial and vascular fibrosis, cardiac arrhythmias, sodium retention and reduced vascular compliance and baroreceptor function.

Thus, direct renin inhibitors prevent angiotensin-I and II formation, with beneficial effects of AT1 blockade and aldosterone inhibition, but with the potentially adverse effects of AT2 inhibition. ACE-i have the beneficial effects of AT1 blockade and aldosterone inhibition, but with the potentially adverse effects that result from inhibiting AT2. In addition, non-ACE pathways can bypass ACE-i, resulting in AT1 stimulation. Furthermore, ACE could potentially have adverse effects because of the AT2 blockade. ACE-i have unique positive cardiovascular effects that result from bradykinin inhibition, which, unfortunately, also causes dry cough (10-30\% incidence), the most frequent side effect. Angiotensin receptor blockers (ARBs) specifically block AT1 and aldosterone production and upregulate AT2, which all have beneficial cardiovascular effects. Aldosterone inhibitors exert positive cardiovascular effects by specifically inhibiting aldosteronemediated adverse effects. ACE-i, ARBs and aldosterone inhibitors result in the upregulation of renin production, which could cause negative cardiovascular effects directly by renin, through pathways which are unknown at present (Figure 1).

\section{CLINICAL EVIDENCE}

All specific effects of the aforementioned RAS-blocking drugs have been tested, with the hypothesis that direct renin inhibition with additional ACE-i and/or ARBs would be most beneficial because of the full RAS blockade. Because of the low bioavailability and difficulties in the production process, and because of the clinical success of ACE- $i$ and later ARBs, renin inhibitors were not used in clinical practice until the 21 st century. Multiple large prospective trials have demonstrated the blood pressure lowering effect of ACE-i and the use of ARBs as RASblocking monotherapy. ${ }^{1-3}$ Therapy with both antihypertensive drug classes resulted in reduced cardiovascular and renal endpoints, such as stroke, myocardial infarction, heart failure, dialysis, deterioration of renal function, albuminuria and the incidence of type 2 diabetes mellitus. Meta-analyses have shown that both drug classes were equally successful in preventing cardiovascular and renal complications, contrary to some individual studies that have suggested more myocardial infarction in ARBs versus ACE-i. In the ONTARGET trial, the combination of 


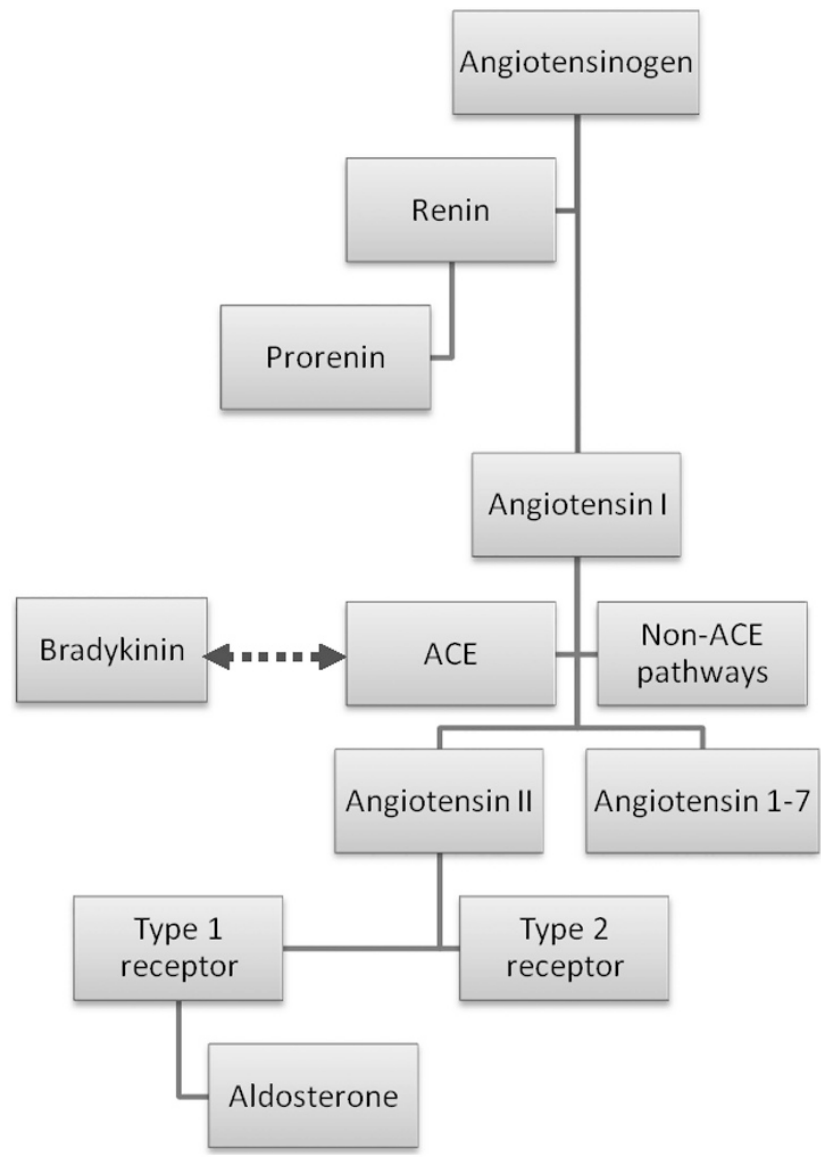

Figure 1 The renin-angiotensin-aldosterone system (RAS). See text for the clinical consequences of the different RAS enzymes and the effects of the drugs on the RAS. ACE, angiotensin-converting enzyme. A full color version of this figure is available at Hypertension Research online.

ARBs (Telmisartan) and ACE-i (Ramipril) resulted in more adverse events, without an increase in benefits for patients with a high risk of cardiovascular disease. In the VAILLANT study, the combination of ACE-i and ARBs did not result in beneficial ventricular remodeling or function after myocardial infarction compared with monotherapy. One study that did show a positive effect was the CHARM-ADDED study. In patients with NYHA class II-IV heart failure and left ventricular ejection fraction (LVEF) $<40 \%$, the combination of candesartan and an ACE-i resulted in significantly lower morbidity and mortality rates. In the ASPIRE and ALLAY trials, the addition of a renin inhibitor was not beneficial in terms of ventricular remodeling in post-myocardial infarction patients. In diabetics, the addition of the renin inhibitor aliskiren to ACE-i or ARBs (ALTITUDE trial) was shown to be disadvantageous by causing more hypotensive episodes and significant hyperkalemia. The early termination of the ALTITUDE trial seems to confirm previous concerns about the safety of the dual pharmacological blockade (for example, renin inhibitors, ACE-i and ARBs) of the RAS in patients. Conversely, the addition of an aldosterone antagonist to standard therapy (that is, the EPHESUS trial), ${ }^{4}$ including beta-blockers and ACE-i or ARBs, has consistently demonstrated a reduced number of cardiovascular death in patients after myocardial infarction with LVEF $<40 \%$ and heart failure symptoms or diabetes.

Thus, the clinical evidence indicates the importance of blood pressure reduction and (strong) RAS inhibition but not by combining different RAS-blocking agents. ACE-i and ARBs have provided far more evidence than direct renin inhibitors in preventing cardiovascular and renal complications, with ARBs being preferable in terms of side effects. Particularly in antihypertensive drug therapy, patient compliance is essential; therefore, ARBs are one of the most appealing antihypertensive drug classes.

\section{AZILSARTAN}

In this issue of the journal, Takagi et al. ${ }^{5}$ present a meta-analysis of the new and potent ARB azilsartan. In 2011, the drug was approved by the US Food and Drug Administration or hypertensive patients. The authors found seven reports of randomized trials of azilsartan, including 6152 hypertensive patients. These trials were published between 2011 and 2013, pointing out the novel character of the drug. A pooled analysis suggested a significantly stronger blood pressure reduction for azilsartan dosages of $40 \mathrm{mg}$ and $80 \mathrm{mg}$ once daily but not for $20 \mathrm{mg}$ compared with the intensive control therapy. Intensive control therapy consisted of olmesartan $4 \mathrm{mg}$ or ramipril $10 \mathrm{mg}$ or olmesartan/hydrochlorothiazide $40 / 25 \mathrm{mg}$ or valsartan $320 \mathrm{mg}$ or candesartan $12 \mathrm{mg}$ or chlorthalidone $25 \mathrm{mg}$ daily dosages. Although there was significant heterogeneity between studies, the authors used the random-effects rather than the fixed-effects model to perform a more conservative evaluation. Furthermore, they performed sensitivity analyses that excluded single trials with similar results. Importantly, the effects of azilsartan were present both for office and 24-h ambulatory blood pressure measurements, the golden standard. In most of the reported trials, the follow-up was relatively short (approximately 24 weeks). It is expected, however, that the antihypertensive effect is sustained. Trials proving the reduction of a hard cardiovascular endpoint with azilsartan therapy are presently lacking.

\section{PLEIOTROPIC EFFECTS OF ANTIHYPERTENSIVES}

Takagi et al., ${ }^{5}$ as have many other researchers, report on the pleiotropic effects of azilsartan in their present meta-analysis. They describe the drug's antithrombotic, antiproliferative and potentially antifibrotic effects and its beneficial effects on insulin sensitivity and glucose metabolism.

The pleiotropic effects of RAS-blocking agents have been discussed thoroughly for both ACE-i and ARBs. ${ }^{3}$ Several small experimental studies have shown blood pressure-independent effects, with beneficial effects on glucose metabolism and reduced albuminuria, reduced atherosclerosis and inflammation, and improved endothelial function. However, it is unclear how to translate these effects into clinical endpoints. Data from meta-analyses point towards a modest effect of both ACE-i and ARBs in terms of blood pressureindependent reductions of heart failure and renal complications. Only one trial has suggested a blood pressure-independent effect on stroke prevention with the ACE-i 
perindopril; however, other studies have failed to show such an effect of ARBs or ACE-i. In a meta-analysis of antihypertensive drug trials, Staessen et al. ${ }^{6,7}$ showed that blood pressure reduction itself is by far the most important prevention against cardiovascular and renal complications and that the pleiotropic effects of antihypertensive drug classes only have little impact. There was a small blood pressure-independent effect of ACE-i on the prevention of coronary heart disease events (particularly heart failure) and of calcium antagonists on the prevention of stroke, whereas calcium antagonists have a small independent effect on a higher incidence of heart failure.

Thus, in patients requiring (antihypertensive) therapy with a RAS-blocking agent, ACE-i or ARBs seem to be indicated without the addition of other RAS-blocking agents. The exception is the addition of an aldosterone antagonist (eplerenone or spironolactone) in patients after myocardial infarction with LVEF $<35 \%$ and NYHA functional class II-IV or LVEF $<40 \%$ and diabetes or heart failure symptoms (class I evidence).

\section{CONCLUSIONS}

In conclusion, RAS-blocking drugs in combination with other drugs are successful in preventing cardiovascular complications, such as heart failure, stroke, cardiac arrhythmias, myocardial infarction, albuminuria and renal failure. The theoretical advantage of combining renin inhibitors (for example, ACE-i and ARBs) did not translate into a clinical advantage and should be avoided. Azilsartan is a novel ARB, with potent blood pressure reduction and beneficial pleiotropic cardiovascular effects. Azilsartan could be an excellent choice for treating hypertension or an alternative to other ACE-i or ARBs when blood pressure goals are not reached.
1 Mansur SJ, Hage FG, Oparil S. Have the renin-angiotensin-aldosterone system perturbations in cardiovascular disease been exhausted? Curr Cardiol Rep 2010; 12: 450-463.

2 Probstfield JL, O'Brien KD. Progression of cardiovascular damage: the role of renin-angiotensin system blockade. Am J Cardiol 2010; 105: 10A-20A.

3 Bakris G. Are there effects of renin-angiotensin system antagonists beyond blood pressure control? Am J Cardiol 2010; 105: 21A-29A.

4 Pitt B, Remme W, Zannad F, Neaton J, Martinez F, Roniker B, Bittman R, Hurley S, Kleiman J, Gatlin M, Eplerenone Post-Acute Myocardial Infarction Heart Failure Efficacy and Survival Study Investigators. Eplerenone, a selective aldosterone blocker, in patients with left ventricular dysfunction after myocardial infarction. New Engl J Med 2003; 348: 1309-1321.

5 Takagi H, Mizuno Y, Niwa M, Goto S, Umemoto T for the ALICE (All-Literature Investigation of Cardiovascular Evidence) Group. A meta-analysis of randomized controlled trials of azilsartan therapy for blood pressure reduction. Hypertens Res 2014; 37: 432-437.

6 Staessen JA, Wang JG, Thijs L. Cardiovascular protection and blood pressure reduction: a meta-analysis. Lancet 2001; 358: 1305-1315.

7 Staessen JA, Li Y, Thijs L, Wang JG. Blood pressure reduction and cardiovascular prevention: an update including the 2003-2004 secondary prevention trials. Hypertens Res 2005; 28: 385-407. 